\section{La mécanique de l'adhésion cellulaire dans la gastrulation du poisson zèbre}

Jean-Léon Maître ${ }^{1,2}$, Hélène Berthoumieux ${ }^{3,4}$, Simon Frederick Gabriel Krens ${ }^{1}$, Guillaume Salbreux ${ }^{4}$, Frank Jülicher ${ }^{4}$, Ewa Paluch ${ }^{5}$, Carl-Phillip Heisenberg ${ }^{1}$

> Au cours du développement embryonnaire, la formation des différents tissus repose sur la capacité des cellules différenciées à s'organiser en couches distinctes [1]. Deux propriétés mécaniques des cellules, l'adhésion et la contractilité cellulaires, sont essentielles à la formation des tissus [2]. Elles contribuent au contrôle de la formation et de la stabilité des contacts entre cellules (Figure IA,B), et des différences dans leur capacité à former ces contacts peuvent induire la ségrégation des cellules en couches distinctes [3-5]. Par conséquent, comprendre les rôles respectifs de l'adhésion et de la contractilité cellulaires dans la formation des contacts intercellulaires est essentiel à notre compréhension de la formation des tissus.

L'adhésion entre les cellules repose sur la formation de liaisons entre des protéines transmembranaires de la famille des cadhérines, qui favorise la croissance du contact entre deux cellules. Au contraire, la contractilité des cellules, sous l'action du cortex d'actomyosine, s'oppose à la déformation du contact entre deux cellules, et tend donc à en réduire la taille. Toutefois, les mécanismes cellulaires gouvernant la formation de ces contacts et coordonnant les propriétés adhésives et contractiles ne sont pas compris.

Nous avons étudié les rôles respectifs de l'adhésion et de la contractilité cellulaires dans la formation des contacts, et ce dans le modèle de la gastrulation du poisson zèbre. La gastrulation est une phase précoce du développement embryonnaire durant laquelle les cellules se différencient en trois feuillets germinaux : l'ectoderme, le mésoderme et l'endoderme, à partir d'une population homogène de cellules. Chez le poisson zèbre, la seule régulation par les cadhérines est insuffisante pour expliquer la formation des feuillets germinaux [2]. Un des avantages de cet organisme modèle est la facilité d'obtention et de manipulation des cellules pour reproduire ex vivo certains processus cellulaires et développementaux. Nous avons tiré parti de ces atouts pour comprendre la séparation des feuillets germinaux en étudiant la mécanique de formation de contacts entre deux cellules.

\section{Mécanique de l'adhésion entre cellules} Nous avons développé un modèle théorique décrivant les forces impliquées dans la régulation de l'angle de contact entre deux cellules (Figure 1B) [6]. Ces forces, qui agissent à la surface de la cellule, prennent la forme de forces par unité de surface, ou tensions de surface. À l'état stationnaire, les tensions de surface résultant de l'action des molécules d'adhésion - $\omega$ (tension d'adhésion)-, du cortex d'actomyosine au contact $-\gamma_{c c}$ (tension corticale du contact) -, et du cortex d'actomyosine hors du contact $-\gamma_{\mathrm{cm}}$ (tension corticale) -, s'équilibrent et déterminent l'angle de contact (Figure IB).

Grâce à deux micropipettes permettant de maintenir en contact deux cellules,
${ }^{1}$ Institute of science and technology Austria, Klosterneuburg, Autriche ;

${ }^{2}$ European Molecular Biology Laboratory, Heidelberg, Allemagne;

${ }^{3}$ Laboratoire de physique théorique de la matière condensée, Paris, France ;

${ }^{4}$ Max Planck institute für physik komplexer systeme, Dresde, Allemagne ;

${ }^{5}$ Max Planck institute for cell biology and genetics, Dresde, Allemagne, et International institute of molecular and cell biology,

Varsovie, Pologne.

maitre@embl.de

nous avons pu former et observer le comportement des doublets de cellules. Nous avons ainsi mesuré des contacts plus importants pour les doublets de cellules ectodermiques que pour les doublets de cellules mésodermiques ou endodermiques. Pour comprendre comment ces différents états stationnaires sont atteints, nous avons développé un modèle théorique décrivant la forme des cellules adhérentes. L'analyse de nos résultats expérimentaux dans ce modèle nous permet de mesurer la tension corticale du contact. Enfin, connaissant l'angle de contact, nous avons pu déterminer la tension d'adhésion (Figure IC,D). Cette étude a montré que, quel que soit le feuillet d'origine, la tension d'adhésion est négligeable par rapport à la tension corticale du contact. Ceci nous permet de conclure que l'angle de contact est principalement déterminé par le rapport des tensions corticales $\gamma_{c c} / \gamma_{c m}$, plutôt que par la tension d'adhésion. Les cellules ectodermiques forment donc des angles de contact plus grands que les angles formés par les cellules mésodermiques et endodermiques, car le rapport des tensions corticales $\gamma_{c c} / \gamma_{c m}$ y est plus bas. C'est par ce mécanisme de modulation locale de la tension corticale au niveau des contacts cellule-cellule que, dans l'embryon, les cellules mésodermiques et endodermiques (qui ont des contacts plus petits), formeraient un feuillet différent du feuillet ectodermique où les contacts entre cellules sont plus grands. 

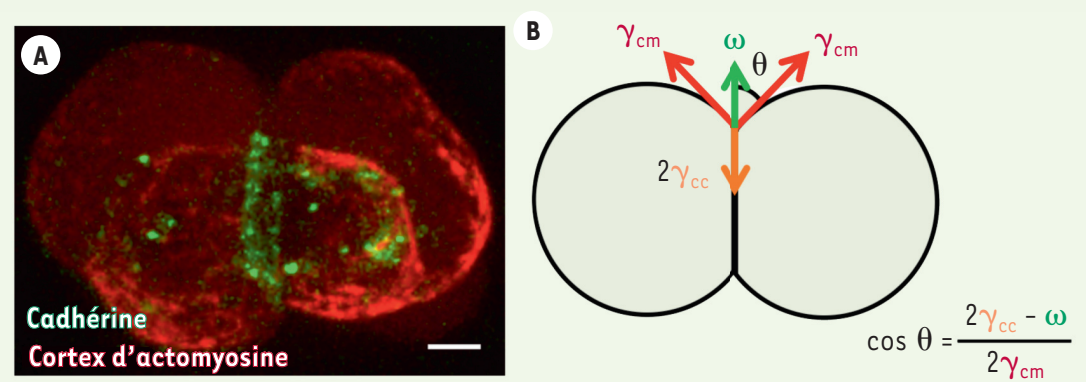

c

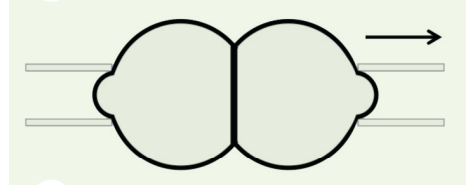

$\varepsilon$ D
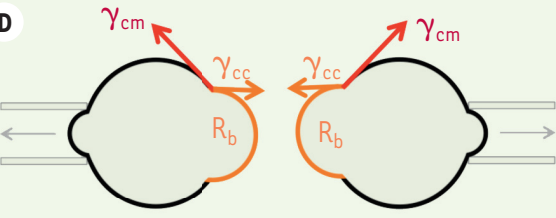

$F_{a}$

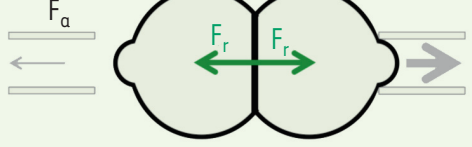

$\mathbf{F}$
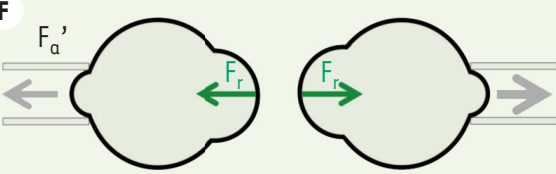

Figure 1. Forces contrôlant la formation d'une adhésion entre cellules. A. Image de deux cellules ectodermiques en contact. La cadhérine (en vert) montre le complexe d'adhésion entre les deux cellules formant un anneau, partiellement visible, et la myosine (en rouge) montre le cortex d'actomyosine. Barre d'échelle : $5 \mu \mathrm{m}$. B. Schéma décrivant les forces (sous forme de tensions de surface) qui contrôlent l'angle de contact $\theta$ entre deux cellules. L'accumulation de complexes d'adhésion entre les deux cellules induit une tension $\omega$ (tension d'adhésion) qui augmente l'angle de contact $\theta$. Le cortex d'actomyosine produit au contact une tension $\gamma_{c c}$ (tension corticale du contact), et à l'interface avec le milieu extracellulaire une tension $\gamma_{\mathrm{cm}}$ (tension corticale). La première tend à diminuer l'angle de contact, la seconde à l'augmenter. À l'état stationnaire, ces forces s'équilibrent et déterminent l'angle de contact $\theta$. C. Schéma de deux cellules en contact tenues par deux micropipettes (en gris) avant leur déplacement visant à séparer les cellules. D. Schéma décrivant le procédé expérimental amenant à déterminer le rapport de tensions corticales $\gamma_{c c} / \gamma_{c m}$. Nous avons séparé des cellules en adhésion et observé la déformation de la surface précédemment en contact (marquée en orange sur la figure). La courbure de cette surface $\left(1 / R_{b}\right)$ ne dépend plus de la tension d'adhésion $\omega$, les liaisons d'adhésion ayant été rompues lors de la séparation. Ceci nous permet de calculer la tension corticale du contact $\gamma_{c c}$ et, connaissant l'angle de contact $\theta$, nous pouvons déterminer la tension d'adhésion $\omega$ (voir panneau $B$ ). $\varepsilon-F$. Schéma décrivant le procédé expérimental amenant à déterminer la résistance mécanique du contact aux forces de séparation $F_{s}$. En appliquant une force d'aspiration $F_{a}$ inférieure à la force de résistance mécanique $F_{r}$ du contact, les cellules restent attachées après le déplacement des micropipettes. En appliquant une force d'aspiration $F_{a}$ ' supérieure à la force de résistance mécanique $F_{r}$ du contact, les cellules se détachent après le déplacement des micropipettes. La force de séparation $F_{s}$ est calculée comme la moyenne de $F_{a}$ et $F_{a}$ '.

\section{Quel rôle mécanique pour} les molécules d'adhésion?

Bien que la tension d'adhésion ait une influence négligeable dans la régulation de la taille du contact entre les cellules, les molécules d'adhésion ont un rôle essentiel dans le maintien des contacts. $\varepsilon n$ particulier, elles permettent de résis- ter aux forces qui pourraient séparer les cellules. Pour comprendre le rôle de la résistance mécanique des molécules d'adhésion dans la formation du contact, nous avons étudié leur capacité à résister aux forces de séparation [7]. Pour cela, nous avons utilisé des micropipettes pour séparer des doublets de cellules et mesurer la force nécessaire à leur séparation (Figure 1E, F). Nous avons mesuré les forces nécessaires à la séparation de doublets composés de deux cellules issues de chaque feuillet germinal, et mis en évidence une plus grande résistance au détachement des cellules ectodermiques par rapport aux cellules mésodermiques ou endodermiques. La taille des contacts, le type de molécule d'adhésion ou leur quantité au site de contact ne suffisent pas à expliquer ces différences dans la force de séparation. Cela suggère l'existence d'un autre facteur de régulation.

Nous avons observé des différences dans l'arrangement des molécules d'adhésion au niveau du contact. Dans le cas de deux cellules ectodermiques, les cadhérines et les autres protéines impliquées dans la formation de liaisons adhésives, comme l' $\alpha$-caténine, forment un anneau. Cette organisation traduit l'existence d'une concentration plus élevée des molécules du complexe d'adhésion à la périphérie du contact. Cet anneau n'est pas observé dans les contacts entre des cellules mésodermiques ou endodermiques (Figure 2A). De plus, durant la séparation, le complexe d'adhésion semble se dissocier, non pas par séparation des cadhérines appartenant aux deux cellules en contact, mais par séparation entre les cadhérines et le cytosquelette d'actine (Figure 2B). Le facteur limitant la résistance mécanique à la séparation se situerait donc au niveau de l'ancrage des cadhérines au cortex d'actomyosine. Ces observations suggèrent que l'attachement des cadhérines au cytosquelette d'actine pourrait varier selon les feuillets. Cette hétérogénéité expliquerait les différences de résistance mécanique au niveau des contacts des cellules dans les différents feuillets germinaux.

Pour tester l'influence du couplage des cadhérines au cortex, nous avons produit une cadhérine mutante qui ne peut pas s'attacher au cytosquelette d'actine [8]. En comparaison des cellules exprimant 

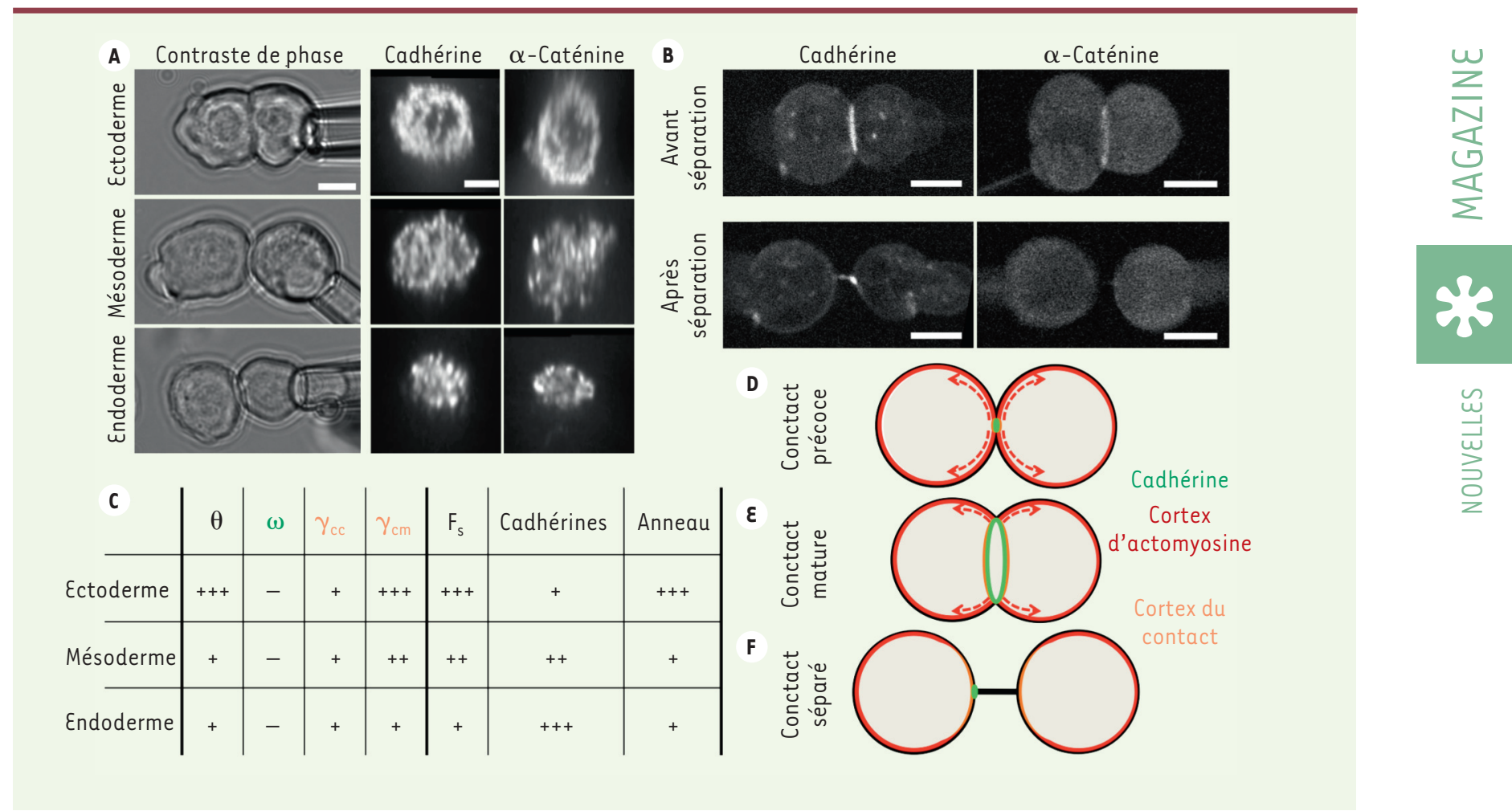

Figure 2. Mécanique de l'adhésion entre cellules germinales du poisson zèbre. A. Images de doublets de cellules ectodermiques, mésodermiques ou endodermiques en contact analysées par contraste de phase perpendiculairement au contact (gauche, contraste de phase, échelle : $10 \mu \mathrm{m}$ ), ou par immunofluorescence dans le plan du contact (droite, cadhérine et $\alpha$-caténine, échelle : $5 \mu \mathrm{m}$ ). Les doublets ectodermiques forment des contacts plus grands que les doublets mésodermiques ou endodermiques (voir panneau $C$ ). Les molécules d'adhésion des doublets ectodermiques forment un anneau qui est moins visible aux contacts des doublets mésodermiques ou endodermiques (voir panneau $\boldsymbol{C}$ ). B. Images de doublets de cellules exprimant une cadhérine ou une $\alpha$-caténine fluorescente avant et après leur séparation mécanique. Durant la séparation, les dimères de cadhérines s'accumulent en un point avant d'être retirés du cortex d'une des deux cellules, alors que l' $\alpha$-caténine se dissocie du contact. Cela suggère que l'ancrage cytoplasmique de la cadhérine, plutôt que son attachement extracellulaire, contribue à la force de séparation $F_{s}$ du doublet. Barre d'échelle : $10 \mu \mathrm{m}$. C. Tableau résumant les propriétés adhésives et contractiles des cellules ectodermiques, mésodermiques et endodermiques. Les tensions de surface $\omega, \gamma_{c c}$ et $\gamma_{c m}$, et l'angle de contact $\theta$, sont présentés dans la Figure IB. La quantité et l'organisation des cadhérines au contact, supposées réguler la force de séparation $F$ s, sont présentées dans le panneau $A$ et la Figure $1 E-F$. D-F. Schéma récapitulatif décrivant la formation et la séparation du contact entre les cellules. Le cortex d'actomyosine à l'extérieur du contact (en rouge) tire sur le complexe d'adhésion (en vert), ce qui, en combinaison avec une réduction de la tension du cortex d'actomyosine du contact (en orange), définit la taille de la zone d'adhésion. Les molécules d'adhésion connectent les cortex d'acto-myosine des deux cellules en contact et résistent au détachement. La résistance mécanique du complexe d'adhésion est limitée par l'ancrage des cadhérines au cortex d'actomyosine.

une cadhérine normale, les cellules exprimant la cadhérine tronquée ne résistent qu'à des forces de séparation plus faibles. $\varepsilon t$ si la force requise pour séparer des cellules ectodermiques exprimant une cadhérine normale est plus grandes que celle qui est requise pour séparer des cellule endodermiques, cette différence est abolie si les cellules expriment la cadhérine tronquée. Ces résultats indiquent que la différence de résistance mécanique des contacts entre cellules ectodermiques et entre cellules endodermiques dépend de l'ancrage des cadhérines au cytosquelette d'actine (Figure 2C).

\section{Conclusions}

Nos résultats indiquent que c'est en modulant la tension corticale au niveau du contact cellulaire que les cellules contrôlent la taille de la zone d'adhésion. Contrairement à ce que l'on croyait au vu de modèles antérieurs, la tension d'adhésion contribue peu méca- niquement à l'étalement du contact cellulaire. Le rôle mécanique principal des molécules d'adhésion serait de connecter les cortex d'actomyosine au points de contact cellulaires. Le complexe d'adhésion doit ensuite résister aux forces qu'exercent les cortex d'actomyosine sur ce contact cellulaire (Figure 2D,F).

Dans l'embryon de poisson zèbre, nous pouvons supposer que c'est en raison de leur moindre capacité à former des 
contacts de grande taille et de grande stabilité que les cellules mésodermiques et endodermiques se séparent des cellules ectodermiques pour former des couches de cellules distinctes. Les principes mécaniques présentés ci-dessus permettent de comprendre les nombreux mouvements morphogénétiques qui impliquent la formation et la stabilité des contacts entre les cellules. $\diamond$

Cell adhesion mechanics of zebrafish gastrulation

\section{REMERCIEMENTS}

Nous remercions J. Compagnon pour ses commentaires. Ces travaux sont financés par la Société Max Planck, le Ministère des sciences et des études supérieures polonais (454/N-MPG/2009/0), le programme scientifique Human Frontier (RGy0067/2008 pour $\varepsilon$. Paluch) et par les Fonds pour la recherche scientifique autrichienne (FWF HE3231/6; 1812-B12 pour C.P. Heisenberg).

\section{LIENS D’INTÉRÊT}

Les auteurs déclarent n'avoir aucun lien d'intérêt concernant les données publiées dans cet article.

\section{RÉFÉRENCES}

1. Krens SFG, Heisenberg CP. Cell sorting in development. Curr Top Dev Biol 2011 ; 95 : 189-213.

2. Krieg M, Arboleda-Estudillo Y, Puech P, et al. Tensile forces govern germ-layer organization in zebrafish. Nat Cell Biol $2008 ; 10: 429-36$.

3. Lecuit T, Lenne P. Cell surface mechanics and the control of cell shape, tissue patterns and morphogenesis. Nat Rev Mol Cell Biol 2007 ; 8 : 633-44.
4. Borghi N, James Nelson W. Intercellular adhesion in morphogenesis: molecular and biophysical considerations. Curr Top Dev Biol 2009 ; 89 : 1-32.

5. Maître JL, Heisenberg CP. The role of adhesion energy in controlling cell-cell contacts. Curr Opin Cell Biol $2011 ; 23: 508-14$.

6. Maître JL, Berthoumieux H, Krens SFG, et al. Adhesion functions in cell sorting by mechanically coupling the cortices of adhering cells. Science 2012 ; 338 : 253-6.

7. Chu Y, Thomas W, Eder 0 , et al. Force measurements in $\varepsilon$-cadherin-mediated cell doublets reveal rapid adhesion strengthened by actin cytoskeleton remodeling through Rac and Cdc42.J Cell Biol 2004 ; $167: 1183-94$.

8. Pacquelet A, Rørth P. Regulatory mechanisms required for $D E$-cadherin function in cell migration and other types of adhesion. J Cell Biol 2005 ; $170: 803-12$.

\section{NOUVELLE}

\section{Un nouvel espoir : greffer des cellules souches dans l'oreille interne}

Jean-Luc Puel
Inserm U1051, Institut des neurosciences, Université de Montpellier, hôpital Saint Eloi, 80, rue Augustin Fliche, 35095 Montpellier, France. jean-luc.puel@inserm.fr
> Les déficits auditifs représentent un véritable enjeu de santé publique. Très récemment, M.N. Rivolta et son groupe (université de Sheffield, Royaume-Uni) [1] ont montré que des progéniteurs neuronaux issus de cellules souches embryonnaires des crêtes neurales humaines peuvent se différencier en neurones auditifs in vivo, et restaurer la fonction auditive dans un modèle de neuropathie chez des animaux adultes. Si la greffe des cellules sensorielles (cellules ciliées) semble encore hors de portée thérapeutique, la transplantation des progéniteurs neuronaux devrait permettre d'élargir l'indication des implants cochléaires aux patients présentant des pertes neuronales [10] $(\rightarrow)$.

$(\rightarrow)$ Voir $m / s$ 2010, vol. 26, $n^{\circ} 11$, page 981
Les enjeux physiopathologiques de la presbyacousie Les statistiques européennes et nord-américaines montrent que $16 \%$ de la population adulte présente des problèmes auditifs. En Europe, le coût de la prise en charge des déficits auditifs représentent 213 milliards d'euros par an, dont 22 milliards pour la France [2]. Dans nos sociétés de plus en plus bruyantes, où l'espérance de vie ne cesse d'augmenter, la surdité liée à l'âge (presbyacousie) devient un véritable enjeu de santé publique. Malheureusement, peu de traitements répondent efficacement aux pathologies de l'oreille interne. Cette pénurie de traitements est en grande partie due au fait que les cellules sensorielles et les neurones auditifs ne se renouvellent pas, ce qui entraîne une surdité irréversible.
Depuis le début du XXI ${ }^{\mathrm{e}}$ siècle, de très nombreux travaux portant sur les cellules souches ont été publiés. Ainsi, la cochlée des mammifères possède des cellules souches (ou progénitrices) ayant la capacité se diviser et de se différencier en cellules ciliées [3]. Lorsque ces cellules souches sont injectées chez l'embryon dans la vésicule otique, elles s'intègrent dans l'épithélium cochléaire et se différencient en cellules sensorielles [4]. En revanche, l'anatomie de la cochlée adulte ne permet pas l'intégration de ces cellules souches au sein de l'épithélium cochléaire [5].

\section{Restauration de la fonction \\ de neurones auditifs in vivo}

Les travaux de l'équipe de M.N. Rivolta récemment publiés dans Nature [1] 